\title{
EXTREME POINTS OF SOME CONVEX SUBSETS OF $L^{1}(0,1)$
}

\author{
JOHN V. RYFF ${ }^{1}$
}

1. Introduction. One characterization of the partial order $\prec$ of Hardy, Littlewood and Pólya for real $n$-vectors is that $y \prec x$ if and only if $y$ belongs to the convex hull $\Omega(x)$ of the set of permutations of $\boldsymbol{x}$. That is, the convex hull of $\left\{P_{\boldsymbol{x}}: P\right.$ a permutation matrix $\}$. The extreme points of $\Omega(x)$ are precisely the permutations of $x$ (see [5] for some interesting generalizations). This partial order generalizes to a continuous version where vectors are replaced by $L^{1-}$ "functions" on the unit interval. The corresponding question concerning extreme points was raised but only partially answered in [7]. We now settle this issue by showing that the conjecture at the end of [7] is indeed correct: Each extreme point of an orbit $\Omega(f)$ must be equimeasurable with $f$. Furthermore, all the extreme points are also exposed points.

Bold-face will be utilized to distinguish between elements of $L^{1}=L^{1}(0,1)$ and their representatives. Thus, $f \in f$ is interpreted to mean that $f$ is a representative of $f$. All variables will be real and $\mu$ will denote Lebesgue measure on $[0,1]$. As in [6], [7], for example, one may define a partial order $\prec$ between certain elements of $L^{1}$ by means of their decreasing rearrangements. If $\boldsymbol{f} \in L^{1}$ we set

$$
m(y)=\mu\{f>y\} .
$$

This defines a nonincreasing, right continuous function on the real line. Denote by $f^{*}$ its right continuous inverse. That is,

$$
f^{*}(s)=\sup _{m(y)>s} y .
$$

Then $f^{*}$ is in tegrable and we denote its class by $\boldsymbol{f}^{*}$. Moreover $\boldsymbol{f}$ and $\boldsymbol{f}^{*}$ have the same integral and

$$
\int_{0}^{8} f \leqq \int_{0}^{8} f^{*}, \quad 0 \leqq s \leqq 1 .
$$

If $g \in L^{1}$ and

$$
\int_{0}^{8} g^{*} \leqq \int_{0}^{8} f^{*}, \quad \int_{0}^{1} g=\int_{0}^{1} f, \quad 0 \leqq s \leqq 1,
$$

Received by the editors August 18, 1966.

1 This work supported by the National Science Foundation, Contract NSF GP5829. 
we say that $\boldsymbol{g}$ is majorized by $\boldsymbol{f}$ and write $\boldsymbol{g} \prec f$. In [7] it is shown that $g \prec f$ if and only if $g=T f, T$ a doubly stochastic operator. In addition to this, we know that the set $\Omega(f)=\{\boldsymbol{g}: \boldsymbol{g} \prec \boldsymbol{f}\}$ is weakly compact and convex. It is called the orbit of $f$ (as the doubly stochastic operators constitute a semigroup). If $\phi$ is a measure preserving transformation of $[0,1]$ into itself and $\phi \in \phi$ then $f^{*} \circ \phi \in \Omega(f)$ and each of these elements is an extreme point of $\Omega(f)$. We now show that all extreme points of $\Omega(f)$ arise in this manner. Two useful preliminary remarks are that $\Omega(f)=\Omega\left(f^{*}\right)$ and that if $g$ is an extreme point of $\Omega(f)$ then so too is $g^{*}$. Our argument amounts to showing that if $g^{*} \neq f^{*}$ and $g^{*} \in \Omega\left(f^{*}\right)$ then $g^{*}$ cannot be extreme. This is equivalent to showing that an element $u \neq 0$ exists such that $\boldsymbol{g}^{*} \pm \boldsymbol{u} \prec f$.

It might seem initially that all one needs to do is to choose $u$ sufficiently small so that $\boldsymbol{g}^{*} \pm \boldsymbol{u}$ is still monotone. A moment's reflection on the Cantor function shows that this need not be possible. One must look more carefully at the relationship between $\boldsymbol{g}^{*}$ and $\left(\boldsymbol{g}^{*} \pm \boldsymbol{u}\right)^{*}$.

2. Proof. At the outset we shall suppress the asterisk and assume that $f$ and $g$ are nonincreasing elements of $L^{1}$ with $g \prec f$. Choose rightcontinuous representatives $f$ and $g$, and suppose that for at least one value $s, 0<s<1$,

$$
\int_{0}^{s} g<\int_{0}^{s} f
$$

Then the set $\{t: g(t)<f(t)\}$ contains a point $t_{0}$ of $(0,1)$ at which $g$ is

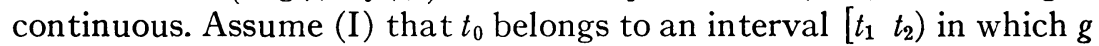
is constant and such that $g$ experiences a jump discontinuity greater than $\eta$ at both end points. If $t_{1}=0$ or $t_{2}=1$ disregard the discontinuity assumption at that point. If $g(s)=c$ for $t_{1} \leqq s<t_{2}$ and

$$
f\left(t_{2}^{-}\right)=\lim _{\boldsymbol{s} \rightarrow \boldsymbol{r}_{2}^{-}} f(s)<c
$$

then for $\alpha>0, \beta>0$ sufficiently small we must have $f(s)<c-\beta$ when $t_{2}-\alpha \leqq s<t_{2}$. In this case choose positive numbers $\delta$ and $\epsilon$ such that $\delta<\min \left(\frac{1}{2}\left(t_{2}-t_{1}\right), \alpha\right)$ and $\epsilon<\min \left(f\left(t_{1}\right)-c, c-f\left(t_{2}^{-}\right), \eta, \beta\right)$. If $f\left(t_{2}^{-}\right) \geqq c$ delete $\alpha, \beta$ and $c-f\left(t_{2}^{-}\right)$from the preceding choices. Define

$$
\begin{aligned}
u(s) & =\epsilon, & & t_{1}<s<t_{1}+\delta, \\
& =-\epsilon, & & t_{2}-\delta<s<t_{2}, \\
& =0, & & \text { otherwise. }
\end{aligned}
$$

Then $(g \pm u)^{*}=g+u \prec f$. To see this observe that 


$$
\int_{0}^{s} f<\int_{0}^{s}(g+u)
$$

could only occur for $t_{2}-\delta<s<t_{2}$ (when $f\left(t_{2}^{-}\right)<c$ ). But the inequality reverses when we put $s=t_{2}$ and yet $f(s)<g(s)+u(s)$ for $t_{2}-\delta<s<t_{2}$ -a contradiction. If $f\left(t_{2}^{-}\right) \geqq c$, then $f>g+u$ in $\left(t_{1}, t_{2}\right)$ and there is nothing to prove. A few obvious modifications will settle the cases where $t_{1}=0, t_{2}=1$ or both.

Suppose now that $g$ is constant in $\left[t_{1}, t_{2}\right), g\left(t_{1}\right)<f\left(t_{1}\right)$ and $g$ is continuous at $t_{1}\left(0<t_{1}<1\right)$. Then $g(s)<f(s)$ in some interval $\left(t_{1}-\delta, t_{1}\right)$ and, since $g \prec f$, we may choose $\delta>0$ in such a way that

$$
\int_{0}^{8} f>\int_{0}^{8} g+\eta
$$

for $t_{1}-\delta \leqq s \leqq t_{1}$ and some $\eta>0$. In the interval $\left[t_{1}-\delta, t_{1}\right]$ select four points $s_{1}<s_{2}<s_{3}<s_{4}$ so that

$$
\begin{aligned}
& g\left(s_{i}\right)>g\left(s_{i+1}\right), \quad i=2,3,4, \quad \text { and } \\
& \int_{0}^{s} f>\int_{0}^{s_{1}} g+g\left(s_{1}\right)\left(s-s_{1}\right), \quad s_{1} \leqq s \leqq s_{4} .
\end{aligned}
$$

If, instead, $g$ has a jump discontinuity at $t_{1}$ (or $t_{1}=0$ ) and is continuous at $t_{2}$ we consider whether or not equality obtains in

$$
\int_{0}^{t_{2}} g \leqq \int_{0}^{t_{2}} f
$$

If the inequality occurs, then we may choose four points $s_{1}<s_{2}<s_{3}<s_{4}$ in an interval $\left(t_{2}, t_{2}+\delta\right)$ such that (3) holds. (Note. One cannot assume that $f \geqq g$ in $\left[s_{1}, s_{4}\right]$.) Suppose equality takes place. Then since $g\left(t_{1}\right)$ $<f\left(t_{1}\right)$, it must be that $g\left(t_{2}\right)>f\left(t_{2}-\right) \geqq f\left(t_{2}\right)$. The inequality continues to the right of $t_{2}$ and the inequality in (2) would reverse. This eventuality may be ruled out.

Finally, we may have a point of continuity $t_{0}$ for $g$ in $(0,1)$ which does not belong to an interval of constancy (where $g\left(t_{0}\right)<f\left(t_{0}\right)$ ). Then it is easy to determine points $s_{1}<s_{2}<s_{3}<s_{4}$ in a neighborhood of $t_{0}$ such that $(3)$ is valid in $\left[s_{1}, s_{4}\right]$. Therefore, if it is impossible to satisfy (I) the following must be applicable.

Propositron. Let $f$ and $g$ be nonincreasing, right continuous and integrable. Assume there exist points $0<s_{1}<s_{2}<s_{3}<s_{4}<1$ such that

$$
g\left(s_{i}\right)>g\left(s_{i+1}\right), \quad \int_{0}^{s} f>\int_{0}^{s_{1}} g+g\left(s_{1}\right)\left(s-s_{1}\right)
$$


for $s_{1} \leqq s \leqq s_{4}$. If $\mathbf{g} \prec \boldsymbol{f}$ then $\boldsymbol{g}$ is not an extreme point of $\Omega(f)$.

Proof. Let $\epsilon=\min \left(g\left(s_{1}\right)-g\left(s_{2}\right), g\left(s_{3}\right)-g\left(s_{4}\right)\right)$ and choose $\delta$ $=\frac{1}{2}\left(s_{3}-s_{2}\right)$. Define

$$
\begin{aligned}
u(s) & =\epsilon, & & s_{2}<s<s_{2}+\delta, \\
& =-\epsilon, & & s_{3}-\delta<s<s_{3}, \\
& =0, & & \text { otherwise. }
\end{aligned}
$$

Then for $0 \leqq s \leqq s_{1}$ and for $s_{4} \leqq s \leqq 1$ we have

$$
(g \pm u) *(s)=g(s) \text {. }
$$

Heuristically, one sees that this should be true by applying the maxim: For any measurable function $h$, both $h$ and $h^{*}$ assume the same values equally of ten. The change from $g$ to $g \pm u$ has no influence on the range of $g$ outside the interval $\left(g\left(s_{1}\right), g\left(s_{4}\right)\right)$. Consideration of the function $m$ defined by (1) and its inverse bears out this assertion. One also must realize that for $s_{1} \leqq s \leqq s_{4},(g \pm u)^{*}(s) \leqq g\left(s_{1}\right)$ from which follows

$$
\int_{0}^{s}(g \pm u)^{*} \leqq \int_{0}^{s_{1}} g+g\left(s_{1}\right)\left(s-s_{1}\right)<\int_{0}^{s} f
$$

when $s_{1} \leqq s \leqq s_{4}$. Lastly since $g \pm u$ and $(g \pm u)^{*}$ are equally distributed in $\left[s_{1}, s_{4}\right]$ they have the same integral, so that

$$
\int_{0}^{s_{4}}(g \pm u)^{*}=\int_{0}^{8_{4}} g \pm u=\int_{0}^{84} g .
$$

Therefore $\boldsymbol{g} \pm \boldsymbol{u} \prec \boldsymbol{f}$ and $\boldsymbol{u} \neq 0$.

3. Exposed points. The reader will recall that the exposed points of a convex set $C$ are defined to be those extreme points through which a closed supporting hyperplane may be passed containing no other points of $C$. If is not difficult to show that every extreme point of $\Omega(f)$ is also an exposed point. Indeed, it will be enough to show that $f^{*}$ is exposed. Enumerate the rational numbers in $(0,1)$ and let $\chi_{n}$ denote the characteristic function of $\left[0, r_{n}\right)$ for each rational $r_{n}$. Set

$$
\psi=\sum_{n=1}^{\infty} 2^{-n} \chi_{n}
$$

Then $\psi$ is nonincreasing, bounded and nonnegative. For any $\boldsymbol{g} \in \Omega(\boldsymbol{f})$ it is known $[4$, p. 62] that 


$$
\int_{0}^{1} g \Psi \leqq \int_{0}^{1} g^{*} \psi \leqq \int_{0}^{1} f^{*} \psi
$$

Consequently, $\psi$ determines a supporting hyperplane for $\Omega(f)$ through $f^{*}$. If equality in (4) held we should have

$$
\sum_{n=1}^{\infty} 2^{-n} \int_{0}^{r_{n}} \xi=\sum_{n=1}^{\infty} 2^{-n} \int_{0}^{r_{n}} g^{*}=\sum_{n=1}^{\infty} 2^{-n} \int_{0}^{r_{n}} f^{*} .
$$

But then $g=g^{*}=f^{*}$ in view of (2). Let $\hat{f}$ be any other extreme point of $\Omega(f)$. Then there exists a measure preserving transformation $\phi$ of $[0,1]$ in to itself such that $\hat{\boldsymbol{f}}=\boldsymbol{f}^{*} \circ \boldsymbol{\phi}$. Set $\hat{\boldsymbol{\psi}}=\boldsymbol{\psi} \circ \boldsymbol{\phi}$. Then for $\xi \in \Omega(f)$

$$
L(g)=\int_{0}^{1} g \hat{\psi} \leqq \int_{0}^{1} g^{*} \psi \leqq \int_{0}^{1} f^{*} \psi=\int_{0}^{1} \hat{f} \hat{\psi}=L(\dot{f})
$$

with equality possible only if $g^{*}=f^{*}$. Now those $h \in L^{1}$ for which

$$
L(h)=\int_{0}^{1} h \hat{\psi}=L(\hat{f})
$$

constitute a supporting hyperplane of $\Omega(\boldsymbol{f})$. If $\boldsymbol{g} \neq \hat{\boldsymbol{f}}$ and $L(\hat{g})=L(\hat{\boldsymbol{f}})$, then $h=\frac{1}{2}(\boldsymbol{g}+\hat{f})$ also lies in this plane. But then $h^{*}=f^{*}$ forcing $h$ to be an extreme point of $\Omega(f)$-a contradiction.

4. Further properties of orbits. Several basically topological features common to all the orbits will be discussed here. First it should be observed that the extreme points of each orbit are closed in the norm topology. This becomes simple once we notice that for measurable functions $f_{n} \rightarrow f$ implies $f_{n}{ }^{*} \rightarrow f^{*}$ (a.e.). Moreover, it is implicit in the work of Brown [2] that the set $\left\{f^{*} \circ \phi: \phi\right.$ invertible measure preserving $\}$ is norm-dense in the extreme points of $\Omega(f)$. A measure preserving transformation $\phi$ is said to be invertible if it is essentially univalent with $\phi^{-1}$ measure preserving. If we go over to the weak topology then the extreme points are dense in $\Omega(f)$. This too follows from [2]. Returning to the $L^{1}$-norm we should mention that the extreme points of $\Omega(f)$ are also dense in themselves, or else $f$ is a constant.

For if $\boldsymbol{f}^{*} \circ \boldsymbol{\phi}$ is such an extreme point ( $\phi$ measure preserving) let $F$ represent any measurable set in which $f^{*}$ is nonconstant. Writing $E=\phi^{-1}(F)$ we select any measure preserving transformation $\pi$ of $E$ into itself such that $\mu\left\{f^{*} \circ \phi \neq f^{*} \circ \phi \circ \pi\right\}>0$. Extend $\pi$ to be the identity on the remainder of $[0,1]$. Then 


$$
\int_{0}^{1}\left|f^{*} \circ \phi-f^{*} \circ \phi \circ \pi\right| \leqq 2 \int_{F}|f|
$$

which can be made as small as we please by choice of $F$.

The preceding two assertions represent a decided break from the finite dimensional case. One question we have been unable to answer is: Are the extreme points of $\Omega(f)$ connected in the norm topology?

A particular reason for our interest in the topological structure of the extreme points of orbits is directly related to the weak compactness of each $\Omega(f)$. The weak topology will be metrizable so that Choquet's theorem applies with no technical difficulty. We doubt, however, that any concrete representation formula (such as the Herglotz formula) can be found in terms of integrals taken over the extreme points. This presumption is based on the preponderance of admissible measures supported by the extreme points. The representation will generally not be unique so that the problem would be one of selecting a distinguished representative for each $\boldsymbol{g} \in \Omega(\boldsymbol{f})$.

In order to obtain probability measures concentrated on the extreme points we note that the extreme points always contain a (norm) continuous image of the unit interval. In particular, if $f^{*}$ is strictly decreasing, the map can be chosen to be a homeomorphism. Thus one can carry all the Borel measures on $[0,1]$ on to the extreme points of $\Omega(f)$. Of course, there may be other measures supported by the extreme points; we simply illustrate one source.

The mapping mentioned above may be obtained by defining a oneparameter family of invertible measure preserving transformations:

$$
\begin{aligned}
\phi_{s}(t) & =\iota(1-s+t), & & 0 \leqq t \leqq s, \\
& =\iota(t-s), & & s<t \leqq 1,
\end{aligned}
$$

where $0 \leqq s \leqq 1$ and $\iota$ represents the identity function $(\iota(t)=t)$. If $f \in L^{1}$ and $s_{1}<s_{2}$ we have with $h=s_{2}-s_{1}$

$$
\begin{aligned}
\left\|f^{*} \circ \phi_{s_{2}}-f^{*} \circ \phi_{s_{1}}\right\|= & \int_{h}^{1}\left|f^{*}(t)-f^{*}(t-h)\right| d t \\
& +\int_{0}^{h}\left|f^{*}(t)-f^{*}(1+t-h)\right| d t .
\end{aligned}
$$

Both integrals tend to 0 with $h$.

There are other, perhaps more suitable, means of representing the elements of $\Omega(\boldsymbol{f})$. For instance, if $g \in \Omega(\boldsymbol{f})$ there will exist a oneparameter family $\left\{\sigma_{s}\right\}$ of probability measures on $[0,1]$ such that 


$$
g(s)=\int_{0}^{1} f d \sigma_{s}
$$

(see $[2, \S 2])$ almost everywhere.

We conclude with some discussion about support points. Recall that the support points of a convex set are those points through which a closed supporting hyperplane may be passed. Since each orbit $\Omega(f)$ lies in the hyperplane

$$
L(\boldsymbol{g})=\int_{0}^{1} g=\int_{0}^{1} f
$$

technically every point of $\Omega(f)$ is a support point. We shall only consider those supporting hyerplanes which do not contain $\Omega(f)$.

Now the support points of any closed convex subset $C$ in a Banach space are norm-dense in the boundary of $C[1$, Theorem 1]. In our setting we can say much more: The support points of each orbit are norm-dense in the orbit.

Suppose then that $g$ is a support point of $\Omega(f)$. Let

$$
L(h) \leqq L(g)=\int_{0}^{1} g \Psi
$$

for some nonconstant $\psi \in L^{\infty}$ and all $h \in \Omega(f)$. Adding a constant to $\psi$ if necessary, we may assume that $\psi \geqq 0$ and write $\psi=\psi^{*} \circ \phi$, with $\phi$ measure preserving. Then

$$
\int_{0}^{1}\left(f^{*} \circ \phi\right)\left(\psi^{*} \circ \phi\right) \leqq \int_{0}^{1} g \Psi \leqq \int_{0}^{1} g^{*} \psi^{*} \leqq \int_{0}^{1} f^{*} \psi^{*}
$$

so that equality must hold. Define

$$
F(s)=\int_{0}^{s} f^{*} \text { and } G(s)=\int_{0}^{s} g^{*}
$$

Then

$$
\int_{0}^{1}(F-G) d \iota^{*}=-\int_{0}^{1}\left(f^{*}-g^{*}\right) \psi^{*}=0 .
$$

The integrand on the left is nonpositive, zero at both end points and $\psi$ is nonconstant. Therefore $F(s)=G(s)$ at least once in $(0,1)$. This shows us that the support points of $\Omega(f)$ are precisely those $g \in \Omega(f)$ such that

$$
\int_{0}^{s} g^{*}=\int_{0}^{8} f^{*}
$$


at least once in $(0,1)$. The sufficiency of this condition should be obvious. Furthermore, (5) will be satisfied by all $g \in \Omega(f)$ if and only if $f$ is a constant. It is enough to note that for nonconstant $f$,

$$
\int_{0}^{s} f^{*}>s \int_{0}^{1} f^{*}
$$

whenever $0<s<1$.

Incidentally, (5) defines a face of the boundary of $\Omega(f)$. The minimal faces then would be those $g$ for which (5) holds for all $s$ in $[0,1]$ the extreme points. If one could show a priori that each extreme point was also a support point, we would have a much shorter argument for describing the extreme points.

Suppose now that $h \in \Omega(f)$ is not a support point. If $h$ is constant $\left(=\int_{0}^{1} f\right)$, define

$$
\begin{array}{rlrl}
g(s) & =\delta^{-1} \int_{0}^{\delta} f^{*}, & & 0 \leqq s<\delta, \\
& =(1-\delta)^{-1} \int_{\delta}^{1} f^{*}, & \delta \leqq s \leqq 1,
\end{array}
$$

for $0<\delta<\frac{1}{2}$. Then $g$ is a nonincreasing element of $\Omega(f)$ which satisfies (5) at $\delta$, and $\|g-h\| \rightarrow 0$ as $\delta \rightarrow 0$.

Assume then that $h$ is not constant and that for at least one sequence $s_{n} \rightarrow 1$ one has $h^{*}\left(s_{n}\right)>h^{*}\left(s_{n+1}\right), n=1,2, \cdots$. Set

$$
\begin{aligned}
\gamma_{n}(s) & =h^{*}(s), & 0 \leqq s<s_{n}, \\
& =h^{*}\left(s_{n}\right), & s_{n} \leqq s \leqq 1 .
\end{aligned}
$$

For some $s_{n}^{\prime}, s_{n}<s_{n}^{\prime}<1$ we should then have

$$
\int_{0}^{s_{n}^{\prime}} \gamma_{n}=\int_{0}^{s_{n}^{\prime}} f^{*}
$$

(since $h^{*}$ and $f^{*}$ have the same integral).

Define

$$
\begin{aligned}
g_{n}(s) & =\gamma_{n}(s), \quad 0 \leqq s \leqq s_{n}^{\prime}, \\
& =\left(1-s_{n}^{\prime}\right) \int_{s_{n}}^{1} f^{*}, \quad s_{n}^{\prime}<s \leqq 1 .
\end{aligned}
$$

One may verify that $g_{n}$ is a nonincreasing support point of $\Omega(f)$ and that $\left\|h^{*}-\boldsymbol{g}_{n}\right\| \rightarrow 0$ as $n \rightarrow \infty$. Finally, write $h=h^{*} \circ \phi$ in the usual way. Then $\boldsymbol{g}_{n} \circ \boldsymbol{\phi}$ will still be a support point and $\left\|\boldsymbol{h} \circ \boldsymbol{\phi}-\boldsymbol{\xi}_{n} \circ \boldsymbol{\phi}\right\|$ 
$=\left\|h-\boldsymbol{g}_{n}\right\|$, completing the argument.

If $h^{*}$ has an interval of constancy in $(\delta, 1]$ with $0<\delta<1$, assume (i) that $h^{*}$ is discontinuous at $\delta$. With $\epsilon=h^{*}\left(\delta^{-}\right)-h^{*}(\delta)$ define for $n=1,2, \cdots$,

$$
\begin{aligned}
\gamma_{n}(s) & =h^{*}(s), & & 0 \leqq s<\delta, \\
& =h^{*}(\delta)+\epsilon / n, & & \delta \leqq s \leqq 1 .
\end{aligned}
$$

There will exist $s_{n}, \delta<s_{n}<1$, such that

$$
\int_{0}^{s_{n}} \gamma_{n}=\int_{0}^{s_{n}} f^{*}
$$

Since $\gamma_{n}$ tends uniformly to $h^{*}$ we must have $s_{n} \rightarrow 1$ as $n \rightarrow \infty$. Let

$$
\begin{aligned}
g_{n}(s) & =\gamma_{n}(s), & 0 \leqq s<s_{n}, \\
& =\left(1-s_{n}\right)^{-1} \int_{s_{n}}^{1} f^{*}, & s_{n} \leqq s \leqq 1 .
\end{aligned}
$$

Again we obtain a sequence of nonincreasing support points of $\Omega(f)$ with $\left\|\boldsymbol{g}_{n}-h^{*}\right\| \rightarrow 0$ as $n \rightarrow \infty$.

If (ii) $h^{*}$ is continuous at $\delta$ (and nonconstant to the left of $\delta$ ) choose a sequence $s_{n} \rightarrow \delta, 0<s_{n}<\delta$, and let

$$
\begin{aligned}
\gamma_{n}(s) & =h^{*}(s), & & 0 \leqq s<s_{n}, \\
& =h^{*}\left(s_{n}\right), & & s_{n} \leqq s \leqq 1 .
\end{aligned}
$$

The remainder of the argument is similar to that in (i).

We might mention that the norm density of the support points is not particular to this setting. It has probably been observed, for example, that the Hilbert cube has this property.

\section{REFERENCES}

1. E. Bishop and R. R. Phelps, The support functionals of a convex set, Proc. Sympos. Pure Math., Vol. 7, pp. 27-35, Amer. Math. Soc., Providence, R.I., 1963.

2. J. R. Brown, Approximation theorems for Markov operators, Pacific J. Math. 16 (1966), 13-23.

3. G. H. Hardy, J. E. Littlewood and G. Polya, Inequalities, Cambridge Univ. Press, Cambridge, 1952.

4. G. G. Lorentz, Bernstein polynomials, Toronto Univ. Press, Toronto, 1953.

5. R. Rado, An inequality, J. London Math. Soc. 27 (1952), 1-6.

6. J. V. Ryff, On the representation of doubly stochastic operators, Pacific J. Math. 13 (1963), 1379-1386.

7. - Orbits of $L^{1}$-functions under doubly stochastic transformations, Trans. Amer. Math. Soc. 117 (1965), 92-100.

UNIVERSITY OF WASHINGTON 